\title{
Synthesis, structural and dielectric properties of 0.8PMN-0.2PT relaxor ferroelectric ceramic
}

\author{
M V TAKARKHEDE ${ }^{1, *}$ and $S$ A BAND ${ }^{2}$ \\ ${ }^{1}$ Department of Physics, J D College Of Engineering and Management (JDCOEM), Nagpur 441501, India \\ ${ }^{2}$ Department of Physics, Yeshwantrao Chavan College of Engineering, Nagpur 441110, India \\ *Author for correspondence (mamta.takarkhed@yahoo.co.in)
}

MS received 17 July 2016; accepted 19 January 2017; published online 29 August 2017

\begin{abstract}
A 0.8PMN-0.2PT solid-solution ceramic was synthesized by columbite processing technique. The effects of sintering temperature on the density, structure and microstructure and in turn on the dielectric properties were investigated. The ceramics sintered at and above $1050{ }^{\circ} \mathrm{C}$ resulted in single-phase perovskite formation. However, high density $>90 \%$ is achieved only after $1170^{\circ} \mathrm{C}$. Microstructural analysis revealed that grain size increases with increase in sintering temperature. A significant increase in the peak of dielectric permittivity only after $1150^{\circ} \mathrm{C}$ owing to increase in density is noted in this study. The quadratic law applied to this ceramic demonstrates that the transition is diffused. The broadness in phase transition and lower dielectric relaxation obtained for the composition demonstrate that the ceramic exhibits characteristics of both relaxor and normal ferroelectrics. The ceramic of composition $0.8 \mathrm{PMN}-0.2 \mathrm{PT}$ exhibits excellent dielectric properties $\varepsilon_{\mathrm{r}-\max }=$ $20294-27338$ at $100 \mathrm{~Hz}$ with $T_{\mathrm{c}}=100-96^{\circ} \mathrm{C}$ at low sintering temperature $1170-1180^{\circ} \mathrm{C}$, respectively.
\end{abstract}

Keywords. Relaxor; dielectric properties; perovskite; pyrochlore; sintering temperature.

\section{Introduction}

Low-firing ceramic dielectrics exhibiting high dielectric constant with low losses are very promising in multilayer capacitors (MLCs). The low firing temperature permits the use of silver or base metals as inner electrodes instead of platinum or palladium, which reduces the cost of MLC [1]. There is a considerable interest in the lead magnesium niobate-lead titanate (PMN-PT) ceramics in MLC and actuator applications due to its high dielectric constant and high electrostatic strain of $0.1 \%$ with low hysteresis. Further, it is an attractive replacement for $\mathrm{BaTiO}_{3}$ in $\mathrm{MLC}$ as it can be sintered at lower sintering temperature than that of $\mathrm{BaTiO}_{3}$, and PZT in actuators as it exhibits high value of dielectric constant at room temperature (RT) [2-6].

It is well known that PT addition in PMN stabilizes the perovskite phase, increases the Curie temperature ' $T_{\mathrm{c}}$ ' and results in improved dielectric properties $[1,2,7,8,12]$. Among the many compositions of PMN-PT relaxor ferroelectrics the composition 0.9PMN-0.1PT and 0.65PMN-0.35PT (MPB composition) are extensively studied [7-26]. In the synthesis of PMN and PMN-PT the formation of unwanted pyrochlore has long been recognized as a major problem [27]. Numerous attempts have been made so far to develop a processing technique to suppress the formation of undesired pyrochlore.

PMN-PT ceramics have been sintered at high sintering temperatures between 1200 and $1300^{\circ} \mathrm{C}$ for the formation of densified product exhibiting enhanced dielectric properties [2,9-11,21,22,24,32,33]. With increasing PT content the solid solution of PMN-PT undergoes gradual transition from relaxor to normal ferroelectric $[7,8,12]$. Various additives such as $\mathrm{MgO}, \mathrm{PbO}, \mathrm{CuO}$ and $\mathrm{ZnO}$ were used in PMN-PT system to get improved dielectric properties at lower sintering temperature [24,28-31].

Very few reports are available on the dielectric properties of the composition 0.8PMN-0.2PT [13,30-32]. In the present work, solid solution of $0.8 \mathrm{PMN}-0.2 \mathrm{PT}$ composition is studied systematically to analyse the effect of sintering temperature on the formation of perovskite phase, densification and microstructure to evaluate the dielectric properties and the nature of phase transition in the low sintering temperature range $1050-1180^{\circ} \mathrm{C}$ for its commercial applications in MLC and actuators.

\section{Experimental}

In the present work, ceramic of composition $0.8 \mathrm{PMN}-0.2 \mathrm{PT}$ is synthesized by the Swartz-suggested double-calcination route. $\mathrm{PbO}$ (99.9\%, Sigma), $\mathrm{MgO}$ (99.9\%, Sigma), $\mathrm{Nb}_{2} \mathrm{O}_{5}$ (99.99\%, Sigma) and $\mathrm{TiO}_{2}$ (99.99\%, Sigma) are used as raw materials. In order to obtain stoichiometric composition the raw oxides are dried separately before weighing. The columbite magnesium niobate $\left(\mathrm{MgNb}_{2} \mathrm{O}_{6}\right)$, designated as $\mathrm{MN}$, is synthesized by mixing of $1.02 \mathrm{~mol} \% \mathrm{MgO}$ and $1 \mathrm{~mol}$ of $\mathrm{Nb}_{2} \mathrm{O}_{5}$. The mixture is milled in an agate mortar using acetone separately. The mixture is calcined at $1050^{\circ} \mathrm{C}$ 
for $3 \mathrm{~h}$. The dried $\mathrm{MN}, \mathrm{PbO}$ and $\mathrm{TiO}_{2}$ are weighed according to stoichiometric formula and ground well with acetone. The homogeneous mass is then calcined in an alumina crucible covered with a lid at calcination temperatures 800 and $850^{\circ} \mathrm{C}$. After calcination the powder is further ground for $20 \mathrm{~min}$. To this homogeneous calcined powder, polyvinyl acetate is added as a binder and the mixture is further ground for 15-20 min. Pellets of 1-2 mm thickness and $1 \mathrm{~cm}$ diameter are then formed by applying uniaxial pressure of 1 ton $\mathrm{cm}^{-2}$ in a stainless-steel die. The pellets are sintered in the closed alumina crucible at temperatures between 1050 and $1180^{\circ} \mathrm{C}$ for $2 \mathrm{~h}$ with a binder burning step at $550^{\circ} \mathrm{C}$. The heating rate during calcination and sintering is $10^{\circ} \mathrm{C} \mathrm{min}^{-1}$ as optimized in the previous study [11]. During the sintering the pellets were covered with the powder of the same composition to minimize the $\mathrm{PbO}$ losses. A detailed flowchart of the synthesis is given in figure 1 .

XRD of sintered ceramics was carried out at RT (Siemens D500 diffractometer, using $\mathrm{CuK} \alpha$ radiation over angular range $2 \theta$ from 10 to $100^{\circ}$ in steps of $0.02^{\circ}$ ). XRD patterns are used to identify the structure formed and to know the relative content of the perovskite phase. The microstructure analysis is carried out using a scanning electron microscopy (SEM) analyser (JEOL JSM84A0). Grain size of the sintered ceramics is determined from the SEM micrographs using a linear intercept method. Densities of the sintered specimens are measured by the Archimedes water displacement method. For electrical measurements the surfaces of the sintered ceramic are polished using silicon carbide sand paper (220, 400 grit) to produce parallel smooth surfaces. Silver paste is coated on both surfaces of the well-polished ceramics and fired at $550^{\circ} \mathrm{C}$ for $30 \mathrm{~min}$ to provide a robust electrode. Dielectric measurements $\left(\varepsilon_{\mathrm{r}}\right.$ and $\left.\tan \delta\right)$ are made using an impedance analyser (Model 4192A Hewlett Packard LF) in the frequency range $100 \mathrm{~Hz}-100 \mathrm{kHz}$ between 30 and $170^{\circ} \mathrm{C}$. The dc resistivity is measured at RT using a picoammeter (Model DPA-111). Current values are measured 2 min after application of voltage $=30 \mathrm{~V}$. The characteristics and dielectric properties of the sintered ceramic are demonstrated in terms of second phase, grain size and density by analysing XRD and SEM.

\section{Results and discussion}

The synthesized ceramics of composition $0.8 \mathrm{PMN}-0.2 \mathrm{PT}$ are designated as reported in table 1 . The dielectric properties

$\left(\varepsilon_{\mathrm{r}-\max }, T_{\mathrm{c}}, \varepsilon_{\mathrm{rRT}}, \tan \delta\right.$ at $T_{\mathrm{c}}$ and RT) and other physical properties such as $\%$ of perovskite phase, lattice constant ' $a$ ', grain size, relative density ' $D$ ', $\Delta T_{\mathrm{c}}$ (difference in $T_{\mathrm{c}, \mathrm{s}}$ from $100 \mathrm{~Hz}$ to $100 \mathrm{kHz}$ ), diffuseness coefficient ' $\delta$ ' and electrical resistivity ' $\rho$ ' of sintered ceramics are summarized in table 2.

The XRD patterns of all ceramics (PMT1-PMT8) are presented in figure 2 . The relative $\%$ of perovskite and pyrochlore phase present in sintered ceramic is calculated from the

\section{First step - preparation of MN}
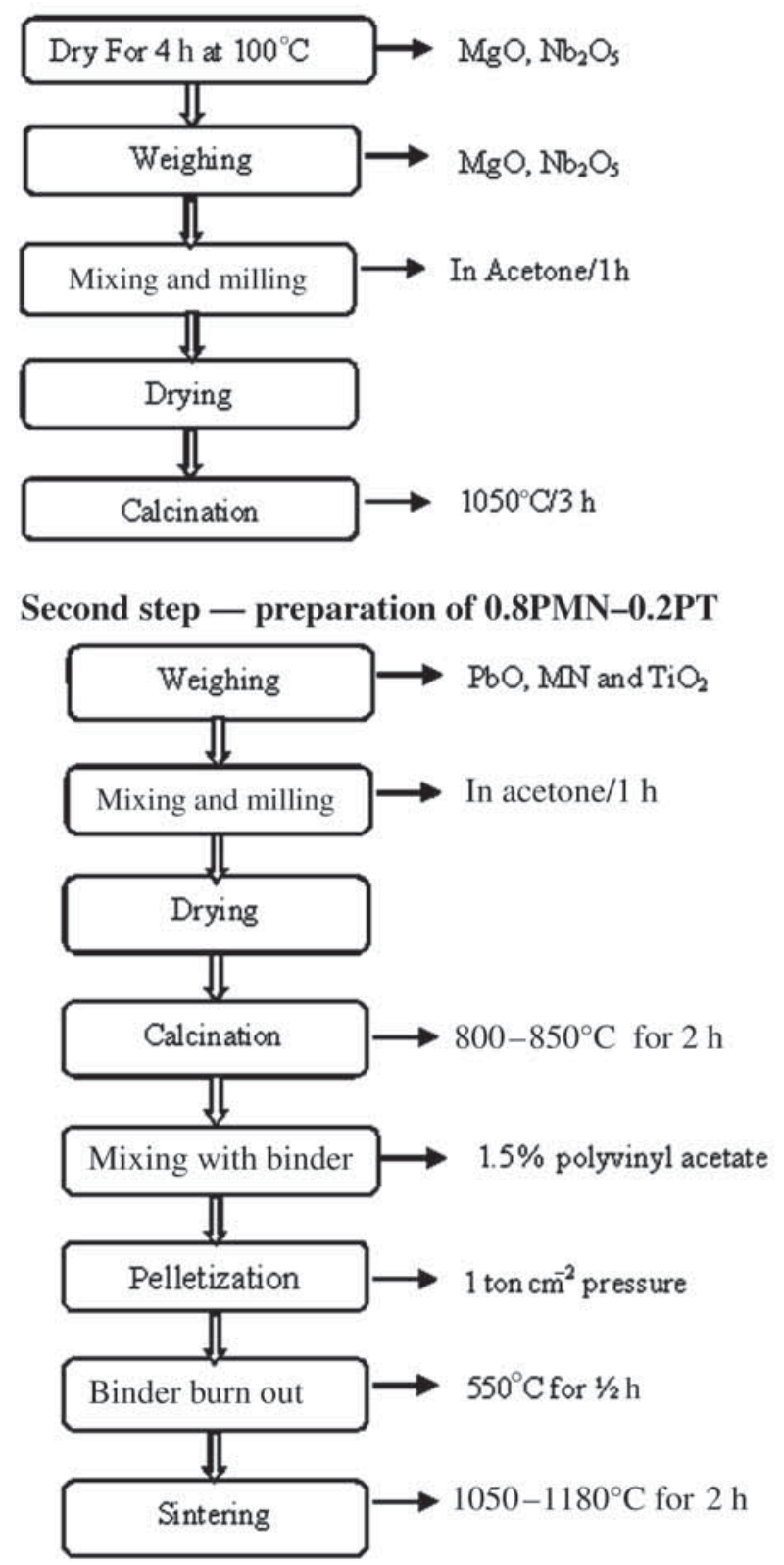

Figure 1. Flowchart of material processing.

intensities of major X-ray reflection for the perovskite (110) and pyrocholre (222) phases using the following equation:

Perovskite $(\%)=\frac{I \text { perov } \times 100}{I \text { perov }+I \text { pyro }}$.

In all the samples (except sample PMT5), the diffraction peaks correspond to pseudo-cubic perovskite phase (JCPDS 271199) and no peaks corresponding to pyrochlore phase appear, which confirms the formation of single-phase perovskite. In the sample PMT5 sintered at $1180^{\circ} \mathrm{C}$, in addition to the perovskite lines, a very small diffraction peak in the region of $29^{\circ} 2 \theta$ is observed, confirming the formation of pyrochlore 
Table 1. Designation of sintered ceramics of composition 0.8PMN-0.2PT.

\begin{tabular}{lcc}
\hline Sample & $\begin{array}{l}\text { Calcination temperature } \\
\left({ }^{\circ} \mathrm{C}\right) \text { and duration }(\mathrm{h})\end{array}$ & $\begin{array}{l}\text { Sintering temperature }\left({ }^{\circ} \mathrm{C}\right) \\
\text { and duration }(\mathrm{h})\end{array}$ \\
\hline PMT1 & 800,2 & 1050,2 \\
PMT2 & & 1100,2 \\
PMT3 & & 1150,2 \\
PMT4 & & 1170,2 \\
PMT5 & 850,2 & 1180,2 \\
PMT6 & & 1050,2 \\
PMT7 & & 1100,2 \\
PMT8 & & 1150,2 \\
\hline
\end{tabular}

phase at $0.3 \%$ therein. This reflects that the decomposition of perovskite phase into pyrochlore just gets initiated at this temperature due to evaporation of $\mathrm{PbO}$, similar to the earlier report [33]. The perovskite phase get stabilized in PMN-PT in the calcination temperature range $800-850^{\circ} \mathrm{C}$, similar to the report by Zhao et al [25]. The lattice constant varies from 3.967 to $3.993 \AA$ in case of ceramics PMT1-PMT5 calcined at $800^{\circ} \mathrm{C}$. With the addition of PT, decrease of the lattice constant is observed, which is attributed to smaller ionic size of $\mathrm{Ti}(0.65 \AA)$ ions, similar to the earlier report $[18,20,32]$. However, for ceramics PMT6-PMT8 calcined at $850^{\circ} \mathrm{C}$ the lattice constant is increased and varies from 4.08 to $4.083 \AA$.

The SEM micrographs of the sintered ceramics are presented in figure 3 . All the ceramics exhibit similar microstructure except the variation of grain size and pore formation. The microstructure reveals increase in grain size and decrease in pore formation with increase in sintering temperature, similar to previous reports $[2,10,30,32]$. At lower sintering temperature, more pores are seen, beside homogeneous distribution of PMN perovskite grains. With increase in sintering temperature, pores decrease and larger variation in grain size is observed. The grain size variation is $1-2,2-3,2.3-3.5,3-4.5$ and 4-6 $\mu \mathrm{m}$ for samples PMT1-PMT5 sintered at 1050, 1100, 1150,1170 and $1180^{\circ} \mathrm{C}$, respectively. Grain size varies from 1-1.7 to $1.5-3.2 \mu \mathrm{m}$ in samples PMT7 and PMT8 sintered at 1170 and $1180^{\circ} \mathrm{C}$, respectively. The ceramics PMT7 and PMT8 calcined at $850^{\circ} \mathrm{C}$ exhibit smaller grains than ceramics PMT1-PMT5 calcined at lower calcination temperature of $800^{\circ} \mathrm{C}$ for the same sintering conditions.

The relative density of the samples is found to increase with increase in sintering temperature. The density of ceramics PMT1-PMT5 sintered at $800^{\circ} \mathrm{C}$ varies from 78 to $95 \%$ as the sintering temperature increases from 1050 to $1180^{\circ} \mathrm{C}$, respectively. However, in samples PMT6-PMT8 calcined at $850^{\circ} \mathrm{C}$, slightly lower density is obtained at the same sintering conditions.

The smaller grain size, slightly larger lattice constant and lower density observed at higher calcination temperature $\left(850^{\circ} \mathrm{C}\right)$ for the same sintering conditions is due to microstructural variation resulting from compositional fluctuations, which seems to be sensitive to calcination temperature.

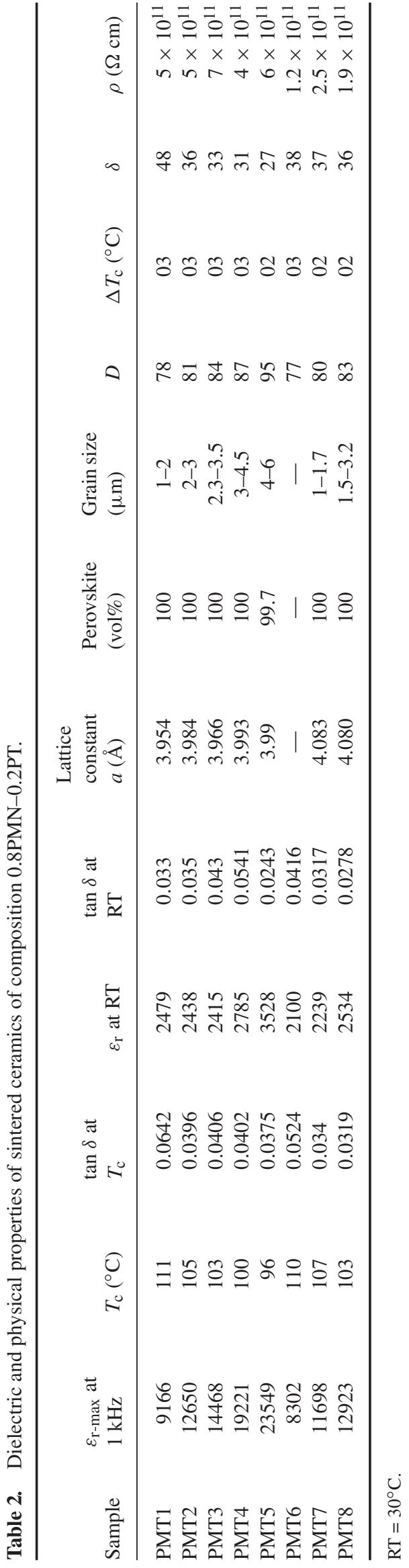


The electrical resistivity of ceramics measured is $>10^{11} \Omega \mathrm{cm}$. Resistivity of the same order was reported by Lejenue and Biolot [34].

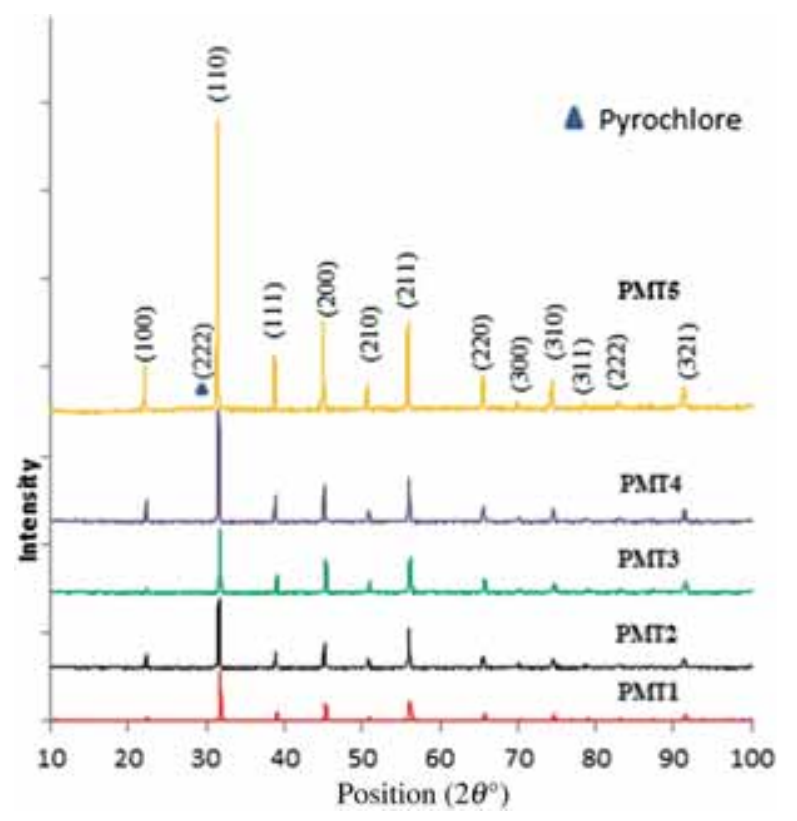

Figure 2. X-ray photographs of sintered ceramics at room temperature.
Figure 4 shows the graph of dielectric constant and the loss tangent $v s$. temperature of typical ceramics PMT2 and PMT5 at four different frequencies $100 \mathrm{~Hz}, 1 \mathrm{kHz}, 10 \mathrm{kHz}$ and $100 \mathrm{kHz}$ in the temperature range $30-170^{\circ} \mathrm{C}$. The peak of dielectric constant decreasing in magnitude as the frequency increases is seen in all samples, demonstrating a typical behaviour of normal or relaxor ferroelectrics. Further, the phase transition is broadened but the shift of $T_{\mathrm{c}}$ with frequency is reduced considerably. A similar trend was reported in previous studies $[8,12,13,19,35]$. The peak of dielectric constant increasing rapidly after sintering temperature $1150^{\circ} \mathrm{C}$ is observed, which seems to be due to increase in density and grain size. The dielectric constant decreases from $T_{\mathrm{c}}$ towards lower temperature, which is due to freezing of domain wall motion and its interaction with lattice defects as the temperature decreases. Increase in frequency leads to decrease in domain wall motion and therefore dielectric constant decreases with frequency, similar to the report by Garcia et al [21]. The transition temperature shifting to the lower side with increase in grain size is observed. A similar transition temperature shift was observed in PMN and PMN-PT systems by other researchers $[2,10]$.

In case of a majority of ceramics the temperature of $\tan \delta_{\max }$, as well as $\tan \delta_{\max }$, increases with frequency. The temperature of $\tan \delta_{\max }$ is lower than that of $\varepsilon_{\mathrm{r} \text {-max }}$ at corresponding frequency, which is a typical behaviour of relaxors.

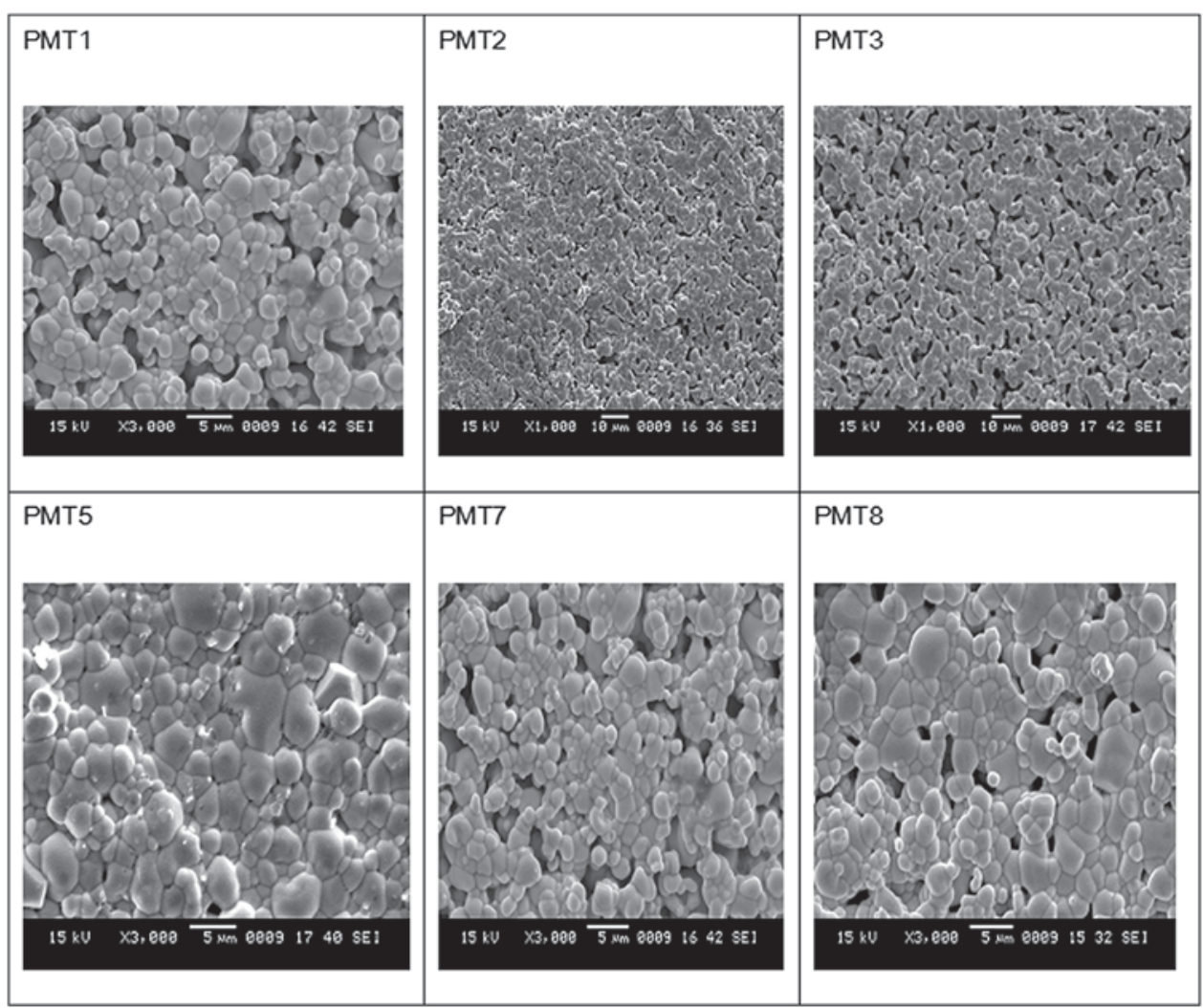

Figure 3. Scanning electron micrographs of sintered ceramics. 

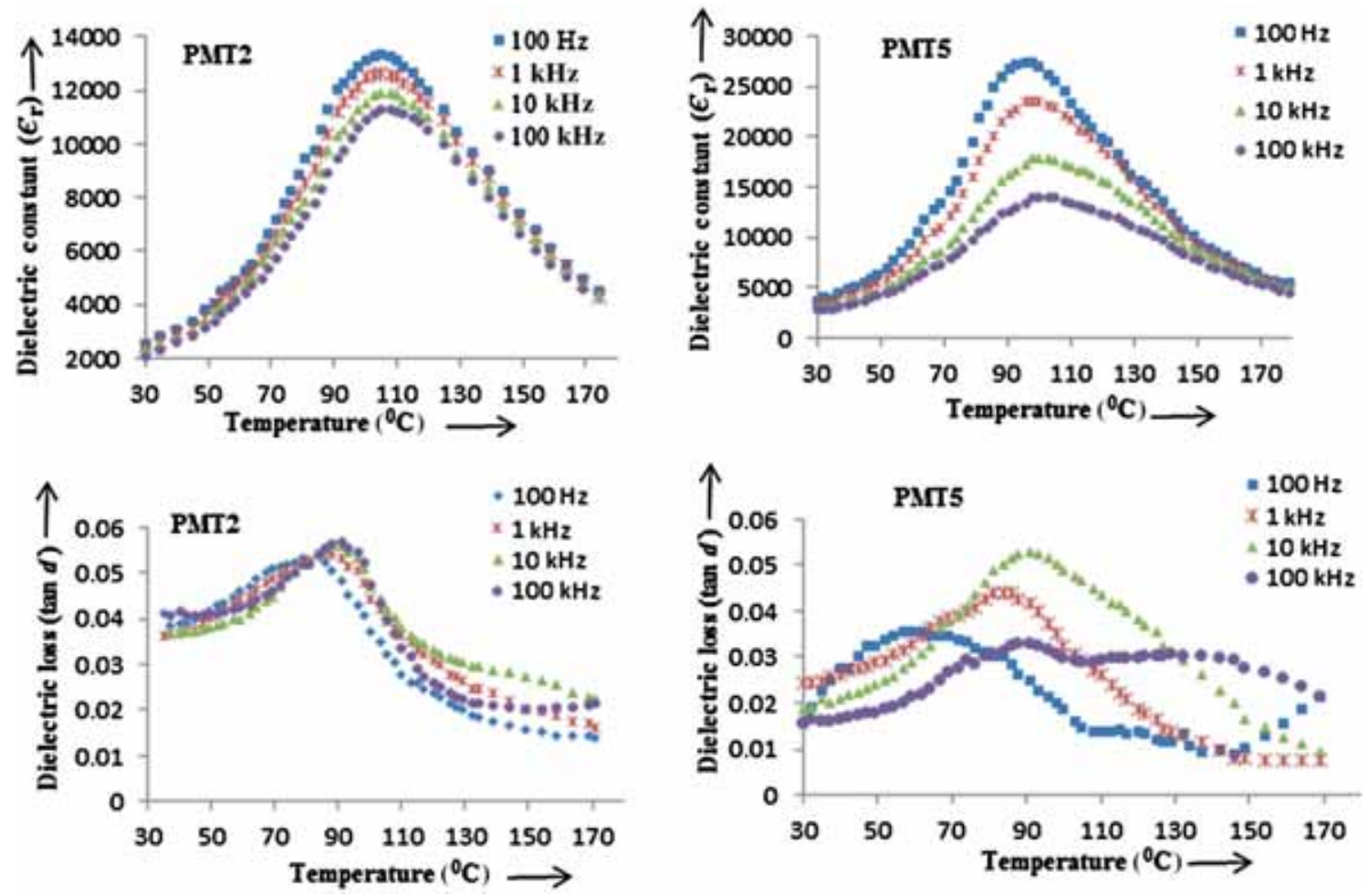

Figure 4. Temperature dependence of dielectric constant and loss tangent of typical sintered ceramics at four different frequencies.

The broadened phase transition obtained is due to micropositional distribution of the $\mathrm{B}$-site cations $(\mathrm{Mg}, \mathrm{Ti})$ in the PMN-PT system, which is presumably due to coexistence of Mg-rich and Ti-rich perovskite phases. The random orientation of these regions causes the structure to be macroscopically cubic but of lower symmetry in the micro-region and thus gives rise to broadened diffraction profile similar to that reported by King et al [5].

The lower dielectric dispersion observed for this composition can be explained in a manner similar to that of prior investigations [12]. For $T>T_{\mathrm{m}}$ the polar clusters are small and do not interact. When the temperature corresponds to $T_{\mathrm{m}}$ their number and size increase. The increase in size allows interactions as the distance between two polar clusters decreases and correlation length becomes longer. Higher the correlation length $L$, smaller the shift of $T_{\mathrm{c}}$ with frequency. Thus the coupling of neighbouring moments implies a lowfrequency relaxation as a result of interactions. The strength of interaction depends on development of polar nanoregions and then on compositions.

The following quadratic law has been used to analyse the relaxor behaviour of solid-solution ceramic $0.8 \mathrm{PMN}-0.2 \mathrm{PT}$ :

$$
1 / \varepsilon_{\mathrm{r}}=1 / \varepsilon_{\mathrm{r}-\max }+\left(T-T_{\mathrm{c}}\right)^{2} /\left(2 \varepsilon_{\mathrm{r}-\max } \delta^{2}\right)
$$

where $\delta$ is called the diffuseness parameter, which is a measure of the diffuseness of transition.

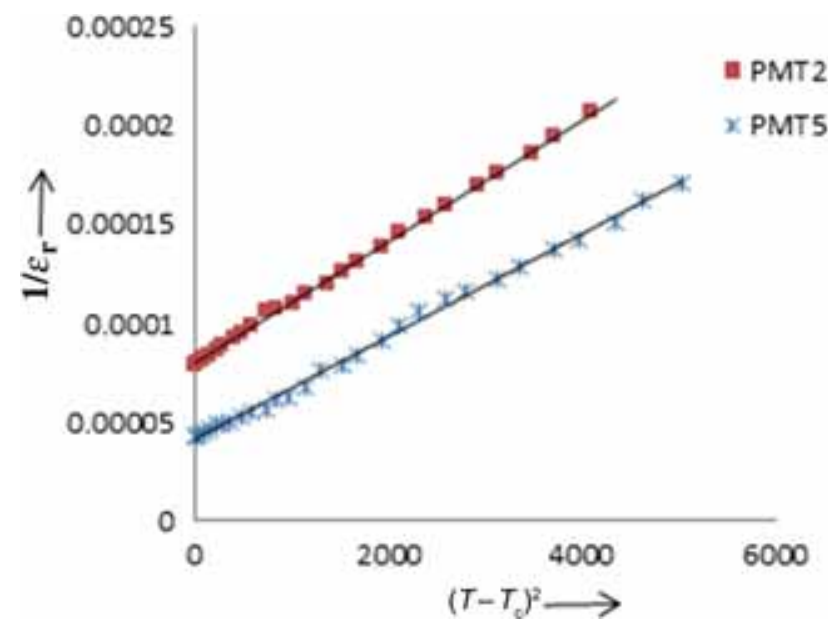

Figure 5. Quadratic graph of typical sintered ceramics.

The graph of $1 / \varepsilon_{\mathrm{r}} v s$. [ $\left(T-T_{\mathrm{c}}\right)^{2}$ for typical samples PMT2 and PMT5 is shown in figure 5. The straight line graph demonstrates that the ceramics exhibit relaxor behaviour. The diffuseness parameter determined from the slope of graph is reported in table 2 . It is observed that $\delta$ decreases as the sintering temperature increases, similar to earlier reports $[2,10]$. This seems to be due to enhanced micropositional fluctuations in PMN-PT ceramics as the sintering temperature increases. 
Table 3. Comparison of dielectric properties of PMN-PT ceramics.

\begin{tabular}{lccccc}
\hline Composition & Sintering temp. $\left({ }^{\circ} \mathrm{C}\right)$ & $\varepsilon_{\mathrm{r}-\mathrm{max}}$ & $T_{\mathrm{c}}\left({ }^{\circ} \mathrm{C}\right)$ & Synthesis method & Reference \\
\hline 0.8PMN-0.2PT (PMT5) & 1180 & 27338 & 96 & Columbite method & Current study \\
0.8PMN-0.2PT & - & 25000 & 102 & Columbite method & 8 \\
0.8PMN-0.2PT & 1150 & 24500 & 60 & Sol combustion method & 13 \\
0.8PMN-0.2PT & 1000 & 6500 & 150 & Columbite method & 31 \\
0.8PMN-0.2PT & 1230 & 18000 & 85 & Reaction sintering method & 33 \\
0.8PMN-0.2PT with 3\% & 1090, & 23612, & 86, & Reaction sintering method & 32 \\
excess CuO & 1120 & 18364 & 90 & & \\
\hline
\end{tabular}

Table 2 demonstrates that the dielectric constant is higher for ceramics PMT1-PMT5 calcined at $800^{\circ} \mathrm{C}$ than samples PMT6-PMT8 calcined at $850^{\circ} \mathrm{C}$ due to higher density achieved and manifestation of larger grain size at the same sintering conditions.

The maximum value of $\varepsilon_{\mathrm{r}-\max }=27338$ and $T_{\mathrm{c}}=96^{\circ} \mathrm{C}$ at $100 \mathrm{~Hz}$ is obtained for ceramic PMT5 sintered at $1180^{\circ} \mathrm{C}$. Addition of PT in PMN increased the transition temperature in PMN, similar to previous reports $[1,7,8,12]$. Decrease of the Curie temperature with sintering temperature is observed and it varies from 111 to 96 at frequency $1 \mathrm{kHz}$ for ceramics PMT1-PMT5 as the sintering temperature increases from 1050 to $1180^{\circ} \mathrm{C}$.

The maximum value of $\varepsilon_{\mathrm{r}-\max }=27338$ at lower sintering temperature $=1180^{\circ} \mathrm{C}$ in spite of very small content of pyrochlore $(=0.3 \%)$ obtained in our study is higher than that reported earlier for this composition. The dielectric values are compared in table 3 .

The maximum density $=95 \%$ reached at temperature $1180^{\circ} \mathrm{C}$ for PMT5 is quite high for this composition. The previous studies showed higher density for the PMN-PT system at sintering temperature greater than $1200^{\circ} \mathrm{C}[12,22]$. The sintering temperature $1050-1150^{\circ} \mathrm{C}$ is deficient for the $0.8 \mathrm{PMN}-0.2 \mathrm{PT}$ ceramics to densify; therefore, relative density of the sintered ceramic is low and dielectric values are lower. With increase in sintering temperature, grain size tends to increase, porosity is gradually eliminated and microstructure becomes dense, which increase the bulk density.

Further, it is revealed in our study that the ceramic of composition $0.8 \mathrm{PMN}-0.2 \mathrm{PT}$ exhibits low dispersive maximum of dielectric constant with frequency, a typical behaviour between relaxor and normal ferroelectric similar to that reported by $[7-9,12,19]$. Sintering at low temperature $=$ $1050^{\circ} \mathrm{C}$ leads to the formation of a single perovskite phase but it exhibits reduced dielectric properties owing to low density = $78 \%$, which is further supported by SEM micrographs. However, a high-density ceramic PMT5 (density $=95 \%)$ obtained at higher sintering temperature $=1180^{\circ} \mathrm{C}$ resulted in significant increase in dielectric properties.

The highest value of $\varepsilon_{\text {r-max }}=27338$ obtained for ceramic sintered at $1180^{\circ} \mathrm{C}$ in spite of the small content of pyrochlore shows that the dielectric properties are found to be strongly dependent on density and the grain size. However, the small content of pyrochlore is not detrimental to dielectric properties, similar to that reported by Gupta and Kulkarni [36]. Further, the study shows that calcination temperature $800^{\circ} \mathrm{C}$ and sintering temperature $1170-1180^{\circ} \mathrm{C}$ are optimized parameters to get enhanced dielectric properties.

The enhanced dielectric properties in ceramics at higher sintering temperature are attributed to high density and increased grain size of single-phase perovskite formed. Higher density indicates that larger volume fraction of ceramic is crystalline perovskite. Larger grain size provides a lower proportion of grain boundaries in the ceramics, which can be detrimental to the dielectric properties. As the grain size increases the number of grain boundaries in series decreases and dielectric constant increases $[2,10,17$, $31,32,36]$.

The high value of relative permittivity and low losses at Curie temperature exhibited by ceramic of composition $0.8 \mathrm{PMN}-0.2 \mathrm{PT}$ sintered at relatively lower temperature $=1170-1180^{\circ} \mathrm{C}$ show that it is a potential candidate in MLCs and actuators.

\section{Conclusion}

Enhanced dielectric properties are obtained for ceramics calcined at $800^{\circ} \mathrm{C}$ and sintered at $1170-1180^{\circ} \mathrm{C}$ owing to single-phase formation, high density and large grain size.

The composition 0.8PMN-0.2PT exhibited a broad transition, which is less dependent on frequency; hence, it demonstrated a mixed behaviour between relaxor and normal.

The ceramic of composition $0.8 \mathrm{PMN}-0.2 \mathrm{PT}$ attained large $\varepsilon_{\mathrm{r}}=27338$ and diffuse maximum of dielectric constant. The lowest loss at low sintering temperature $=1180^{\circ} \mathrm{C}$ shows that it is a promising candidate for use in MLCs and actuators.

The relative density and grain size increase with sintering temperature and thus lead to increase in dielectric constant, as revealed. The dielectric properties are found to be strongly dependent on density and the grain size. However the small content of pyrochlore does not dilute the dielectric properties seriously, as revealed in this study. 


\section{References}

[1] Yan M F, Ling H C and Rhodes W W 1987 J. Mater. Res. 4 930

[2] Shrout T R and Halliyal A 1987 Am. Ceram. Soc. Bull. 66704

[3] Cross L E 1996 Mater. Chem. Phys. 43108

[4] Uršič H, Hrovat M, Holc J, Santo Zarnik M, Drnovšek S, Maček S et al 2008 Sens. Actuators B 133699

[5] King H W, Ferguson S H, Waechter D F and Prasad S E 2002 Proceedings of the 2 nd Canada-US CanSmart Workshop, Montreal, Quebec, Canada p 11

[6] Yamashita Y 1994 Jpn. J. Appl. Phys. 334652

[7] Wongmaneerung R, Yimnirun R and Ananta S 2009 J. Mater. Sci. 445428

[8] Hilton A D, Randall C A, Barber D J and Shrout T R 1989 Ferroelectrics 93379

[9] Chen J H and Liou Y-C 2004 Ceram. Int. 30157

[10] Ravindranathan P, Komarneni S, Bhalla A and Roy R J 1991 J. Am. Ceram. Soc. 742996

[11] Liou Y-C and Wu L 1994 J. Am. Ceram. Soc. 773255

[12] Elissalde C, Ravez J and Gaucher P 1994 Mater. Sci. Eng. B22 303

[13] Sekar M M A and Halliyal A 1998 J. Am. Ceram. Soc. 81 380

[14] Park J-H and Kim Y 1998 J. Korean Phys. Soc. 32 S967

[15] Babooram K, Tailor H and Ye Z-G 2004 Ceram. Int. 301411

[16] Ghasemifard M, Hosseini S M and Khorrami G H 2009 Ceram. Int. 352899

[17] Tailor H N, Bokov A A and Ye Z-G 2011 IEEETrans. Ultrason. Ferroelectr. 581920

[18] Shrout T R, Chang Z P, Kim N and Markgraf S 1990 Ferroelectr. Lett. 1263
[19] Zuo R, Granzow T, Lupascu D C and Rodel J 2007 J. Am. Ceram. Soc. 901101

[20] Yu S, Huang H, Zhou L and Ye Y 2008 J. Am. Ceram. Soc. 91 1057

[21] Garcia J E, Guerra J D S, Araújo E B and Perez R 2009 J. Phys. D Appl. Phys. 42115421

[22] Zhai H-F, Tang R-L, Dong A, Guo H-R, Xia Y-D and Wu D 2009 J. Am. Ceram. Soc. 921256

[23] Zhao S, Li Q, Feng Y and Nan C 2009 J. Phys. Chem. Solids 70639

[24] Zhang Y C, Yang Z Z, Ye W N, Lu C J and Xia L H 2011 J. Mater. Sci. Mater. Electron. 22309

[25] Zhao S-X, Li Q, Song F, Li C and Shen D 2007 Key Eng. Mater. 336-338 10

[26] Lam K H, Li K and Chan H L W 2005 Mater. Res. Bull. 40 1955

[27] Swartz S L and Shrout T 1982 Mater. Res. Bull. 171245

[28] Guha J P, Hong D J and Anderson H U 1988 J. Am. Ceram. Soc. 71 C-152

[29] Yoon K H, Ahn K P and Cho Y S 1993 Ferroelectrics 14657

[30] Sharma Y, Sil A and Yadav K L 2005 Indian J. Eng. Mater. Sci. 12317

[31] Fang B, Jiang N, Ding C, Du Q and Ding J 2012 Phys. Status Solidi A 209254

[32] Ding C, Fang B, Du Q and Zhou L 2010 Phys. Status Solidi A 207979

[33] Shaikh P A and Kolekar Y D 2012 J. Anal. Appl. Pyrolysis 93 41

[34] Lejenue M and Biolot J P 1985 Mater. Res. Bull. 20493

[35] Xu G, Luo H, Wang P, Ql Z and Yin Z 2000 Chin. Sci. Bull. 45 1380

[36] Gupta S M and Kulkarni A R 1996 J. Eur. Ceram. Soc. 16473 